\title{
Check valve slam analysis in pumping station
}

\author{
D. Himr ${ }^{\mathrm{a}}$, V. Habán, and P. Dokoupil
}

Brno University of Technology, Faculty of Mechanical Engineering, Energy Institute, Victor Kaplan Department of Fluid Engineering, Technická 2, 616 69, Brno, Czech Republic

\begin{abstract}
Pumping station supplies water for technological process. The check valve in the station was replaced with a new one. The regular test of black out discovered the high pressure pulsations accompanied with noticeable pipeline movement of discharge pipe. It was caused by late check valve closing, probably, when the back flow reached the highest possible velocity. This statement was supported with analysis of results of pressure measurement near the check valve and with a numerical simulation of the flow in the pipeline system.
\end{abstract}

\section{Introduction}

Check valve is a necessary part of any pipeline delivering liquid from lower to upper place. It is usually placed immediately behind the pump. Its resistance should be low, when liquid flows in a positive direction and no reverse flow should be possible. It is important to choose an appropriate construction to get optimal performance of the check valve. There are many different constructions and each one has its advantages and disadvantages. For example, [1] compared properties of basic types (figure 1 to 4 ).

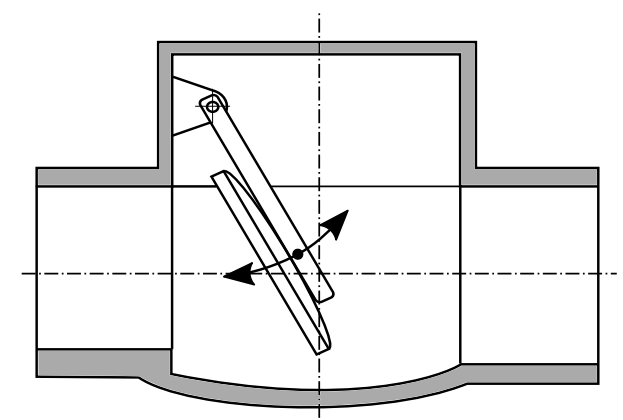

Fig. 1. Swing check valve

The ideal check valve is light with low inertia and low friction loss so it is easy to open it. The disc should have a short run or the closing should be accelerated (e. g. with springs).

There is a practice to slowdown the disc movement to avoid the water hammer, when it is not possible to close disc fast enough when flow reverses. Unfortunately, one has to accept a certain back flow in such case.

According to [2], following properties should be taken into account, while choosing the check valve:

- Closing speed with respect to duration and magnitude of the reverse flow,

- disc stability and its sensitivity to pressure pulsations,

- flow rate necessary to full opening and soft closing,

\footnotetext{
a e-mail: himr@fme.vutbr.cz
}

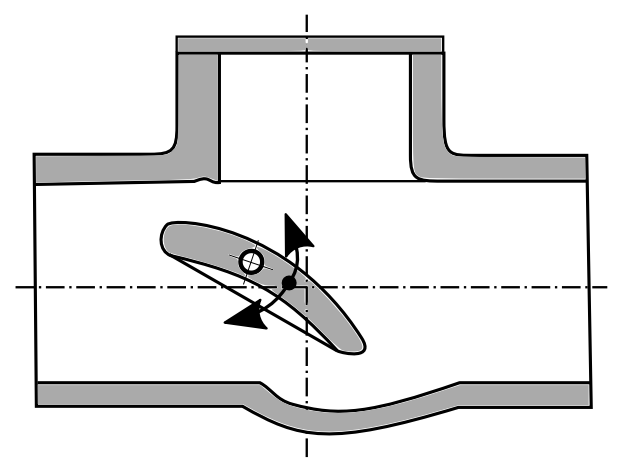

Fig. 2. Tilting check valve

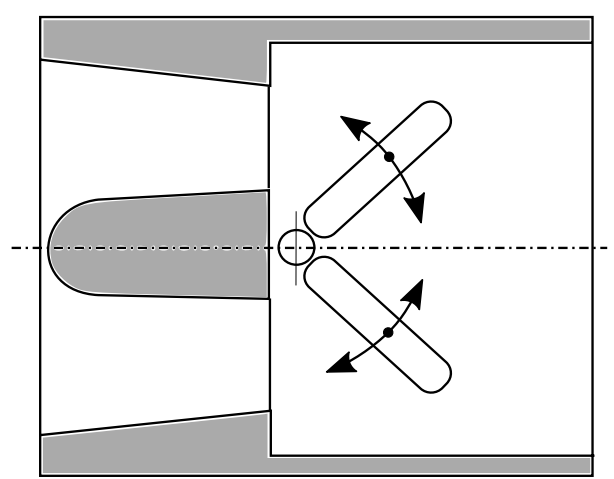

Fig. 3. Split disc check valve

- pressure loss for maximal flow rate,

- tightness and maintenance difficulty.

It means that check valve behaviour depends, besides its construction, on the pipeline system. Choice of a wrong dimension or a wrong type leads to pressure pulsations and check valve slam, which influences lifetime of the check valve and the whole pipeline system. Problems with check valve slam are very common in many industrial applications ([3] or [4]), also authors of this paper have already dealt with it [5]. The valve suffered from self-excited pressure pulsations, but in that case, the problems were not caused by check valve itself, but control valve in the different place of the system. 


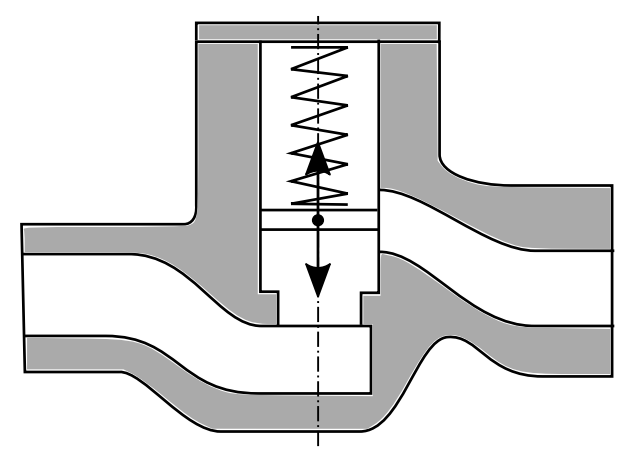

Fig. 4. Lift check valve

Pressure pulsations described in the present paper are consequence of wrong behaviour of the check valve.

\section{Investigated system}

Pumping system delivers water to a technological process, which depends on the continuous supplement of this liquid, see figure 5. One pump delivers water from suction

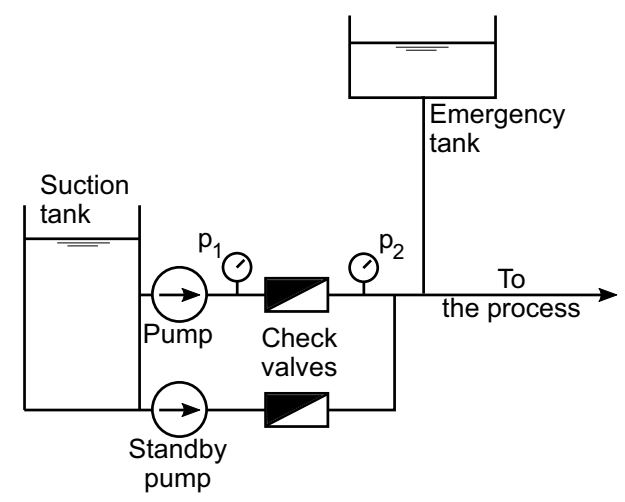

Fig. 5. Investigated system

tank to the discharge pipe. Since the technological process requires a continuous supply, there is another pump ready to start in case of failure of the first unit. Emergency tank connected to the discharge pipe is placed high enough to provide water using the gravity. It gives the time to start the standby pump. Pumps alternates in regular periods to secure the same wearing. Diameter of the discharge pipe is almost $1 \mathrm{~m}$ and when pump runs the steady flow velocity is $1.8 \mathrm{~m} \mathrm{~s}^{-1}$.

Back flow is prevented by check valves near to pumps, they have similar construction to figure 2. There are two counterweights on the shaft to make closing easier, see figure 6 . These counterweights are placed symmetrically at both ends of the shaft.

There are also slide valves between pumps and check valves, but they are not drawn in the figure, because they are open and do not take part in any operating or transient event. These valves are closed just in case of pump revison, reconstruction or similar.

The tests of the system showed strong pressure pulsations accompanied with mechanical vibrations of the delivery pipe. The strength of pulsations led to destruction of operating pressure sensors. Computer interpreted loosing

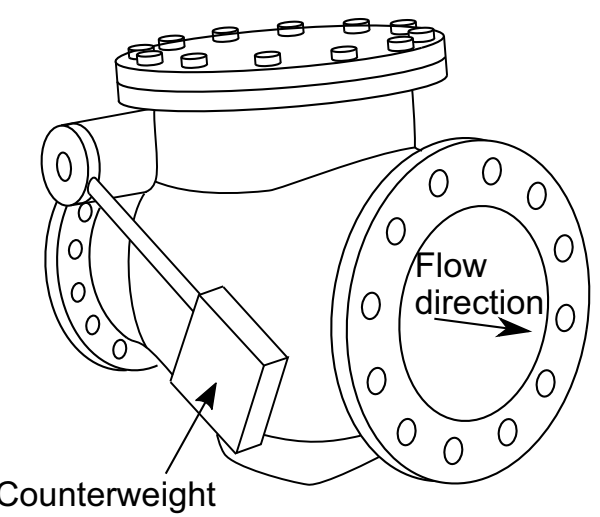

Fig. 6. Counterweight placement

the pressure signal as a pump failure and stopped all process. It was the first indication, that something was wrong.

The system was equipped only with pressure sensors with low sampling frequency. They are sufficient to find out whether the system works or not but they are unable to catch events faster than seconds. So the operator just replaced damaged sensors, but they did not have any suspicion that the problem was deeper. The new sensors failed again after several months.

The problems appeared after the replacement of check valves delivered by different manufacturer than the original ones. The manufacturer stated it had happened because of the wrong operation and it was not their problem, then stopped communication.

The operator took the check valve out of the system and found out it was damaged by the strong slams. The friction of the shaft made closing difficult. The damaged check valve was replaced with a new one from the store. Also this one had quite high friction of the shaft. The following test proved that slams were still on.

Operator tried to install dampers, but they were destroyed after several cycles as well. The operator had a theory that the check valve closed too early and fast when the flow reversed and asked for proving it, but in that case the pressure peak should be much lower than measured one. Description of the measurement follows in the next section.

\section{Measurement evaluation}

\subsection{Sensors}

Measurement conditions were quite difficult, because there is no flowmeter in the system and only two pressure sensors were allowed: downstream and upstream the check valve, see the figure 5. It was also possible to use accelerometer on the pipe wall at the check valve and the strain gauge to get actual disc position. Placement is shown in the figure 7. Range of pressure sensors was 0 to $5 \mathrm{Mpa}$ with error $\pm 0.5 \%$ from the range. Disc position was measured with error $0.2 \%$. This error is for measurement of slow motion or steady position. When faster event is observed the error is greater. 


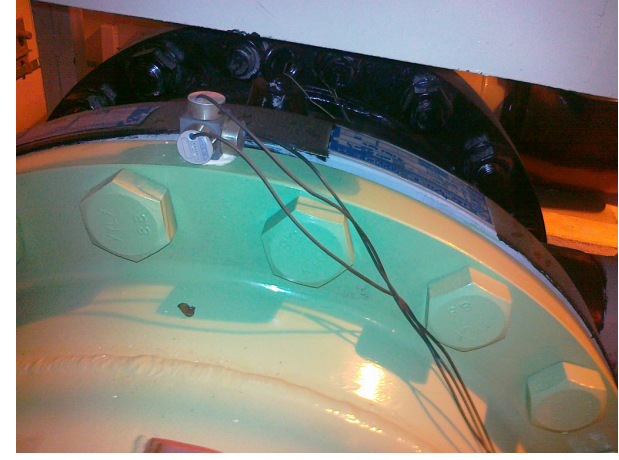

Fig. 7. Accelerometer placement

\subsection{Test}

Regular test starts when pump runs in steady conditions. The whole event is displayed in the figure 8 . The pump was

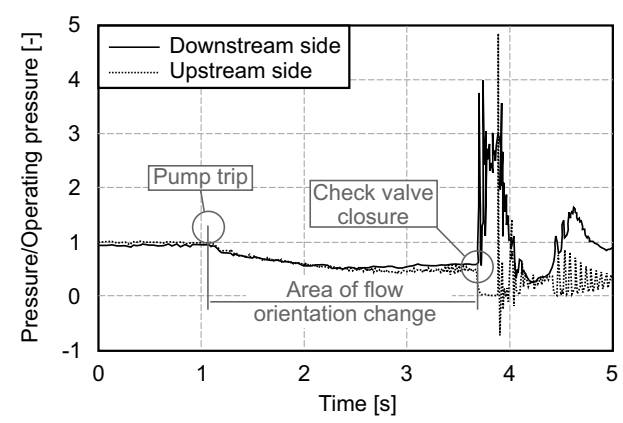

Fig. 8. Pressure measurement at the check valve

disconnected at time $1 \mathrm{~s}$. Pressure went down in both observed places (upstream and downstream side of the check valve) the flow decelerated and reversed. The check valve closed after $2.8 \mathrm{~s}$, which was connected with high pressure surge and strong slam.

The standby pump started running in $15 \mathrm{~s}$ after the the main pump disconnection so it could not influenced the measurement and it is not shown in the graph. Graph in the figure 9 shows what happened after the check valve closure. Point 1 indicates time when check valve started clos-

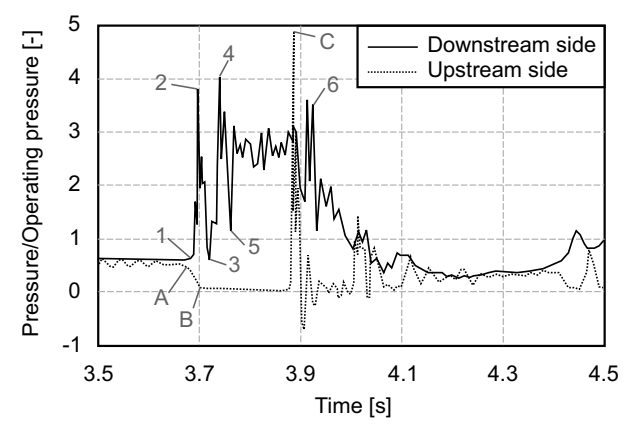

Fig. 9. Pressure measurement at the check valve - detail

ing. The end of closing corresponds to the maximal pressure downstream the check valve (point 2). Sudden drop to the point 3 is not connected with eigen frequency of the system, which is much lower, but when the disc hit the seat, it was bounced and partially opened and closed again (point 4). The bounce was repeated once more (point 5). Typical shock wave, which was induced when the check valve suddenly stopped the flow, travelled to the upstream tank and came back to the check valve. Point 6 marks the moment when the shock wave arrived and pressure dropped down.

The statement regarding the bounce of the disc of the check valve is supported with the graph in the figure 10 . The position of the disc was measured with a position sen-

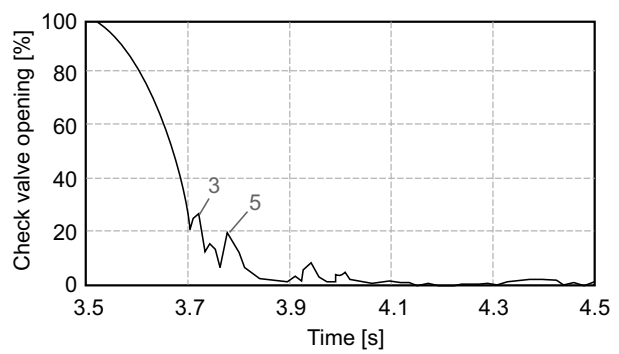

Fig. 10. Check valve opening

sor. This measurement is rather informative, because the sensor had quite great integrating constant, but it shows short periods when the disc was opening (point 3 and 5 in the figure 10 , which correspond to the same points in the figure 9).

In fact, the disc started closing earlier than anything is visible on the pressure record, but its movement had the same velocity as the flow, so it did not make any pressure loss before the full closing.

Figure 9 also shows pressure upstream the check valve. Curve from A to B describes the disc closing, which is followed by cavitation formed at the check valve. Interruption of the back flow caused column separation. Collapsing cavitation is connected with high pressure peak (point C). Negative values do not have physical meaning. Pressure sensors might have been influenced by strong mechanical vibration of the pipe wall, which had acceleration $20 \mathrm{G}$ at that moment, see figures 11 to 13 displaying records of the acceleration sensor at the check valve. Noticeable pipe-

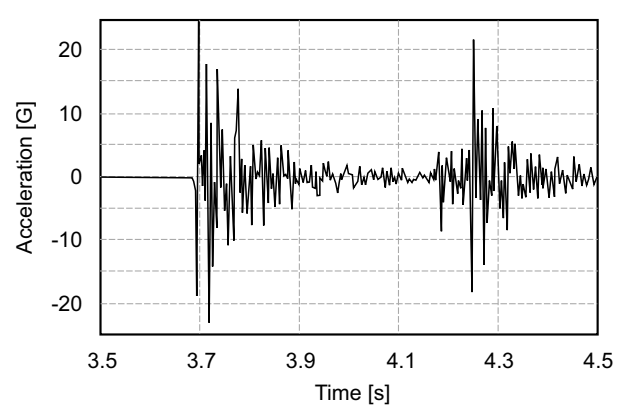

Fig. 11. Wall acceleration in axial direction

line movement was observed during the test and also first signs of damages in the building were observed. Specifically, ruptures in the wall, where the pipe line is going through, were found. 


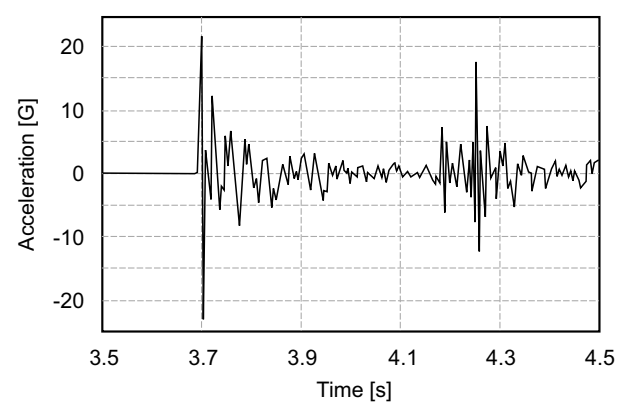

Fig. 12. Wall acceleration in radial (vertical) direction

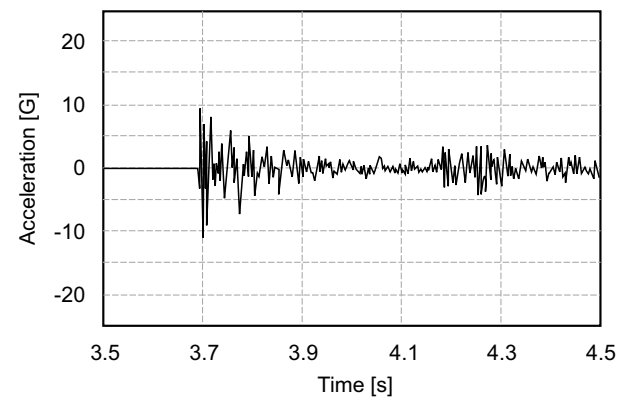

Fig. 13. Wall acceleration in tangential (horizontal) direction

\subsection{Backflow}

Pressure surge is always caused by sudden change in flow rate. There is no flow rate measurement in this case and it is not even possible to use Gibson method to compute time dependence of the flow rate, because the only pressure sensors were at the valve. When the valve closes, it splits the system into two independent parts and each part has only one pressure sensor, but it is possible to estimate back flow velocity using Zhukovsky equation (1).

$$
\Delta v=\frac{\Delta p}{a \cdot \rho} .
$$

Pressure surge $\Delta p$ can be taken from the pressure record downstream the valve. It is the difference between points 2 and 1 (figure 9). Wave speed can be computed from period of pressure pulsations after the fifth second. The check valve was closed and the standby pump was not running yet. The wave speed can be evaluated from both pressure records ( $p_{1}$ and $\left.p_{2}\right)$, see figure 5 and equation (2), which is valid for simple pipe with asymmetric boundary conditions (pressure boundary condition at the one side and flow rate boundary condition at the other side).

$$
a=4 \cdot f \cdot L \text {. }
$$

The back flow velocity can be computed also from the record obtained with pressure sensor upstream the check valve. The process is more complicated than in case of Zhukovsky equation and it is described in [6]. Authors did extensive experiments with cavitating water hammer. Important inputs are initial pressure (point $\mathrm{A}$ in figure 9) and duration of cavitation (time between points B and C). Both approaches give similar value of back flow velocity $1.60 \mathrm{~m} \mathrm{~s}^{-1}$ and $1.52 \mathrm{~m} \mathrm{~s}^{-1}$ respectively, which are too great to say the check valve works in good way.

\section{Computation}

A numerical model of the system has been created to better understand the system behaviour after the pump trip. It is a standard numerical model of one-dimensional flow, where all elements are described with an appropriate mathematical equation.

Look at the figure 5. Suction tank, emergency tank and downstream end of the discharge pipe are conditions of constant pressure. In fact, the emergency tank starts emptying after the pump trip, but observed period is short and it is possible to neglect water level change in this tank. Pump is described by $\mathrm{Q}-\mathrm{p}$ and $\mathrm{Q}-\mathrm{M}$ curves, so, it is possible to simulate pump deceleration and loss of the head. The other pump does not work and its branch is not considered in the simulation, because it is short and does not have any influence on the event.

The check valve is considered as resistance so there is certain pressure loss (3) in the positive direction (direction of flow when pump is running) and backflow is not allowed.

$$
\Delta p=R \cdot|Q| \cdot Q, \forall Q>0 .
$$

Thus, when no solution with positive flow rate is found the flow rate is set to zero and appropriate pressure is computed.

One-dimensional flow in the pipeline follows momentum equation (4) and continuity equation (5). These equations are solved with Lax-Wendroff method.

$$
\begin{array}{r}
\frac{\partial Q}{\partial t}+\frac{S}{\rho} \cdot \frac{\partial p}{\partial x}+\frac{\lambda}{2 \cdot D \cdot S} \cdot|Q| \cdot Q=g_{p} \cdot S, \\
\frac{\partial p}{\partial t}+\frac{K}{S} \cdot \frac{\partial Q}{\partial x}=0 .
\end{array}
$$

As the model has to catch also cavitation at the valve, the dependence of wave speed on the pressure is used [7]. It comes from water - air mixture, where only little air is considered. The air is solved by the gas state equation (6) and it has great impact on wave speed.

$$
\frac{p}{\rho}=r \cdot T
$$

When pressure goes down, the wave speed goes down as well. In an extreme case it can go near to zero.

The result of pump trip numerical simulation does not show any significant pressure fluctuation, see figure 14 . Pressure at the check valve decreases when pump loses

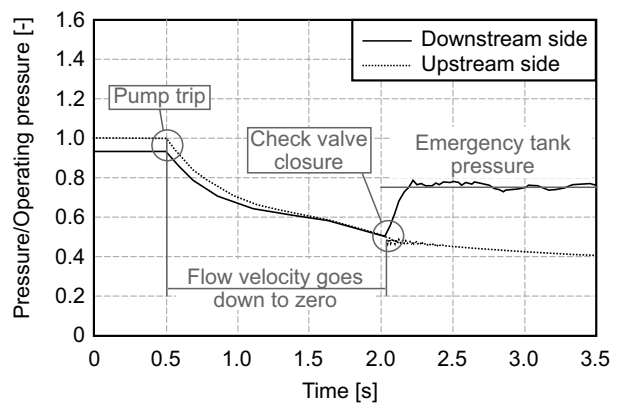

Fig. 14. Pump trip simulation without backflow through the check valve

energy. As soon as flow rate reaches zero value the check 
valve closes and pressure at the downstream side increases to value given by emergency tank. No pressure pulsations appear and there is not any cavitation at the upstream side. Obviously, the result does not correspond to the measurement. It is a confirmation that check valve does not work correctly. The simulation predicts check valve closing approximately $1.5 \mathrm{~s}$ after the pump trip, but measured time is more than two times greater. Compare it with figure 8 .

Figure 15 shows result with adjusted check valve condition. In this case the check valve allows back flow for

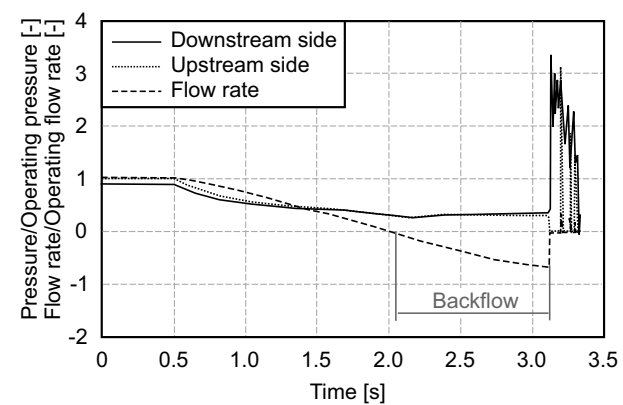

Fig. 15. Pump trip simulation with no backflow through the check valve

certain time and then suddenly closes (the dashed line in the graph). It causes pressure surge (solid and dotted line) fairly corresponding to the measured one. The back flow velocity when the check valve closes is $1.7 \mathrm{~m} \mathrm{~s}^{-1}$. This value fairly correspond to the velocities obtained from pressure measurement analysis described in the previous chapter.

The model of the disc used in the simulation is the simplest one. Inertia, friction or damping are not considered and water does not influences disc behaviour thus the disc bouncing is not present in the result.

\subsection{Discussion}

Simulation showed that the system is designed well and pressure surge cannot appear with correctly working check valve (figure 14). Despite this fact, measured pressure surge indicates great backflow, which has almost the same velocity like the flow when pump is running. It is proved with three independent tools: Analysis of the pressure downstream the valve, upstream the valve and numerical simulation of the pump trip.

It leads to advice to replace the check valve with a different one made by another producer. The temporary solution is to increase the counterweight, which makes closing easier so the backflow will not evolve or it will evolve much lower. This adjustment increases pressure loss, but the pump has enough reserve to secure sufficient flow rate.

It should be stressed, that this solution is temporary. Increased counterweight causes greater strain in the shaft and could lead to its damage as well as the slams.

\section{Conclusion}

This paper describes investigation of real problem with the check valve slam. It appears in a pumping system when the pump loses power supply and it causes noticeable pipeline moving, which is definitely going to serious damage of the system. The problems appeared after the check valve replacement with a new one made by different producer.

The analysis of pressure records upstream and downstream the valve showed that the valve closes when too great back flow evolves. It is too late and it causes strong water hammer. The same result was obtained from results of numerical simulation of flow in pipeline system during the pump trip. The simulation also proved that the system is well designed and it can work without any problem, when good check valve is installed.

To increase the weight of the valve is a temporary solution, which makes its closing easier. The increased pressure loss of the valve due to this modification is acceptable, because the pump is able to cover it and secure the required flow rate.

\section{Acknowledgement}

This work has been supported by Technology Agency of the Czech Republic under the project Innovative research of check valves for extreme operating conditions in energetics TH01011352.

\section{Nomenclature}

\begin{tabular}{|c|c|c|}
\hline$a$ & $\left(\mathrm{~m} \mathrm{~s}^{-1}\right)$ & wave speed, \\
\hline$D$ & $(\mathrm{~m})$ & diameter, \\
\hline$f$ & $(\mathrm{~Hz})$ & frequency, \\
\hline$g_{p}$ & $\left(\mathrm{~m} \mathrm{~s}^{-2}\right)$ & $\begin{array}{l}\text { projection of gravitational accelera- } \\
\text { tion to pipe axis, }\end{array}$ \\
\hline$K$ & $(\mathrm{~Pa})$ & bulk modulus, \\
\hline$L$ & (m) & pipe length, \\
\hline$M$ & $(\mathrm{~N} \mathrm{~m})$ & torque, \\
\hline$p$ & $(\mathrm{~Pa})$ & pressure, \\
\hline$Q$ & $\left(\mathrm{~m}^{3} \mathrm{~s}^{-1}\right)$ & flow rate, \\
\hline$R$ & $\left(\mathrm{~kg} \mathrm{~m}^{-7}\right)$ & resistance, \\
\hline$r$ & $\left(\mathrm{~J} \mathrm{~kg}^{-1} \mathrm{~K}^{-1}\right)$ & gas constant, \\
\hline$S$ & $\left(\mathrm{~m}^{2}\right)$ & pipe cross-section, \\
\hline$T$ & $(\mathrm{~K})$ & temperature, \\
\hline$t$ & (s) & time, \\
\hline$x$ & (m) & longitudinal coordinate, \\
\hline$\Delta p$ & $(\mathrm{~Pa})$ & pressure difference, \\
\hline$\Delta v$ & $\left(\mathrm{~m} \mathrm{~s}^{-1}\right)$ & velocity change, \\
\hline$\lambda$ & & coefficient of friction loss, \\
\hline$\rho$ & $\left(\mathrm{kg} \mathrm{m}^{-3}\right)$ & density. \\
\hline
\end{tabular}

\section{References}

1. K. L. McElhaney, Nuclear Engineering and Design 197, $169-182(2000)$

2. D. Y. Goswami, The CRC Handbook of Mechanical Engineering, Second Edition (CRC Press, 2004), pp. 2688

3. C. Chiu, American Nuclear Society 54, 289 - 291 (1987)

4. J. R. Travis, M. D. Torrey, American Society of Mechanical Engineers winter annual meeting, 1 - 9 (Miami 1985) 
5. D. Himr, V. Habán, Applied Mechanics and Materials 630, 375-382 (2014),

6. R. Autrique, E. Rodal, A. Sánchez, L. Carmona, IOP Conference Series: Earth Environmental Science 15 022014 (2012)

7. D. Himr, Journal of Hydraulic Engineering 141 $04014080(2015)$ 